\title{
Emotional Intelligence and Work Life Balance.
}

\author{
P.Shylaja ${ }^{1}$, Dr. Ch.Jayasankara Prasad ${ }^{2}$ \\ 1. Associate Professor, Aditya Global Business School, Suramplaem, East Godavari Dist., AP \\ ${ }^{2}$ Sr. Asst Prof. of Marketing and HOD I/c Dept. of Business Management, Krishna University, Machilipatnam.
}

\begin{abstract}
Globalization and speed of information and technology instead of making life easy is creating a problem of information overload and thereby pressures in the work place and in work life balance. Emotional Intelligence is a set of skills usually referred to as soft skills or interpersonal or intrapersonal skills that are not covered under the traditional ares of knowledge. Emotions being a part of our genetic makeup we cannot do away wit them even in the workplace.and so are a part of behavior at home or at the workplace.Emotional Intelligence consists of five factors : Knowing ones emotions, motivating oneself, recognizing emotion in others, and handling relationships. Goleman 1995[1]. Therefore it has become imerative for researchers to find the effects of emotional intelligence on employees and organizations. In organizations when it comes to employee management, Work Life Balance has become a challenge for many Managers, Leaders, and Organizations.Emotional intelligence therefore plays an important role in an employee having agood work life balace and benefitting both himself and the Organization.
\end{abstract}

Keywords: Emotional Intelligence, Work life Balance. Managers, satisfaction, organization.

\section{Introduction}

Globalization, overload of Information and communication and Technology have given rise to a very serious problem in the life of a working professional i.e Work Life Imbalance. Emotional Intelligence and Work Life balance are two concepts of high interest in the modern work environment. Especially in the present environment they serve as a competitive edge in Individual and Organizational Life. Also in this century rapid changes in working environment, multi cultural diversity, changes in demography have made it difficult for the average person to adopt and sustain in the global scenario.

\section{Emotional Intelligence}

Emotional intelligence is a part of the genetic makeup of an individual but as with any intelligence this too can be developed. E I improves individual and organizational performance. It plays a significant role in the work of an employee, and the relationship he or she enjoys in the organization. Emotional Intelligence (EI) describes the ability, capacity, skill or, a self perceived ability to identify, assess, manage and control the emotions of one's self, of others, and of groups. Emotional intelligence is considered to play a crucial role in the modern work life. Its principles help in evaluating employee behavior, management styles, attitudes, interpersonal skills and potential. It is also considered to have great relevance in areas like job profiling, planning, recruitment and selection [2,3] Another major advantage of emotional intelligence is that it allows people to better understand and mange emotions. It also helps in understanding one's own conduct as well as relationship with others. Psychological studies have shown that understanding and controlling emotions play significant role in gratifying one's life and work environment.[4,5]

\section{Work Life Balance}

"Work-life balance is the term used to describe those practices at the workplace that acknowledge and aim to support the needs of employees in achieving a balance between the demands of their family (life) and work lives" [6]. Work Life Balance is a challenging issue for managers in all sectors and at all levels of one's career and therefore has also attracted the attention of researchers. Work life balance, in its broadest sense, is defined as a satisfactory level of involvement or fit between the multiple roles in a person's life. In this competitive climate, managing the boundary between home and work is becoming more challenging. This line is even thinner in the some industries, like the IT industry, where the norm now is to work from home and come to the office only when it is absolutely necessary.

Competition in the global scenario has forced Organizations to not only ensure but encourage a practical and workable work life balance, meeting the needs of both the organization and its employees. A major A major reason for attrition at the highest levels of management is because of the inability of the employee to have a work life balance. Organizations not providing real opportunity for employees work/life balance are opening themselves up to increasing numbers of dissatisfied and unproductive employees and hence increased attrition rates.[7] Merely creating a work/life policy framework is not enough; fostering an organizational 
culture that supports the use of available policies is also of great importance. Further there is a need for employers and employees alike to find flexible and innovative solutions that maximize productivity without damaging employees well being, their family relationships and other aspects of life.

This study is an attempt to contribute afresh with a new perspective to the field of human resources and behavioral sciences with special reference to emotional intelligence in relation of employees work life balance in organizations.

\section{Emotional Intelligence And Work Life Balance}

It is generally considered that employees with higher emotional intelligence will have higher work life balance. This is because the employees with higher emotional intelligence are able to cope with consequences which may arise out of stress whereas those with less emotional intelligence won't be in a position to overcome the stress situations. In addition, in a group setting employees with higher EI will be able to influence the emotions of others in such a manner that, they will be able maintain a satisfied personal and professional life.

This paper tries to bring into focus the major challenges encountered in these areas and the solutions that will aid organizations to deal more significantly in increasingly their effectiveness. The review on the related literature in the area of emotional intelligence and work life balance studied in this research has provided the researcher an insight into many factors.

\subsection{Method}

This study uses a descriptive method where in "data is collected to test the hypothesis or to answer questions concerning the current status of the study [8]. A survey method was selected for the present study. The sample consisted of 55 employees from an Organization. The sample consisted both male and female employees. Judgmental sampling technique was adopted to select the number of respondents across the three types of management level.

\subsection{Procedure}

Data Sources: Both primary and secondary sources. The study was conducted among the employees of a manufacturing firm operating in India. Data was collected from 55 employees of the firm using self administered questionnaires.

\subsection{Data Analysis}

Emotional Intelligence questionnaire has five dimensions and is developed by Wong Chi Sum (2007) The questionnaire was based on different dimensions to establish ones EQ : Knowing one's emotions, some of the questions were as follows to be answered on the 5 point likert scale of-Completely agree to..... completely disagree.

\subsection{The major findings of the study were:}

i. Knowing oneself majority of respondents were not completely aware of their capacities and emotions

ii. Managing Emotions a lot of employees were not clear on how to manage emotions at work and home.

iii. Motivating Oneself, Self awareness and realization of one's goals and motivating oneself to achieve those goals in terms of relationships at work and also home.

iv. Recognizing emotions in others, Being able to recognize the power of one's emotions and also the power of influencing others close to you. Being able to understand why people behave differently in various situations is also EI

v. Handling relationships, finally being able to handle relationships at work and home successfully also is being EI. People with higher EI are able to manage both work related relationship with supervisor, Boss, subordinate, and at home with spouse, parents, friends in a successful manner.

vi. The above dimensions were used in the questionnaire to test employees EI

Table -5.1

\begin{tabular}{|l|l|}
\hline Variable & No of items \\
\hline Knowing one's emotions & 6 \\
\hline Managing Emotions & 6 \\
\hline Motivating oneself & 6 \\
\hline Recognizing emotions in others & 6 \\
\hline and Handling relationships & 6 \\
\hline Total & 30 \\
\hline
\end{tabular}


Table -5. 2 The CronBach's Alfa test results were as follows

\begin{tabular}{|l|l|}
\hline $\mathrm{k}$ & 30 \\
\hline Evar & 34.76374 \\
\hline var & 173.918905 \\
\hline Cronbach's Alpha & 0.827705412 \\
\hline
\end{tabular}

Cronbach's alpha, the most widely used objective measure of reliability was used to measure internal consistency. Internal consistency describes the extent to which all the items in a test measure the same concept or construct and hence it is connected to the inter-relatedness of the items within the test. As the test has a reliability of 0.82 , there is 0.33 error variance (random error) in the scores $(0.82 \times 0.82=0.67 ; 1.00-0.67=$ 0.33 ). As the estimate of reliability increases, the fraction of a test score that is attributable to error will decrease. The number of questions on EI were 30 with a variance of 173.918905 and the E var 34.76374.

Work life Balance was tested on another set of 5 questions relating to satisfaction in Pay and benefits, Connection between pay and performance, work load, flexibility in working hours, relationship with supervisors and Boss.

Table 5. -3 Weighted Average Preferences

\begin{tabular}{|l|l|l|l|l|l|l|l|}
\hline \multirow{2}{*}{$\begin{array}{l}\text { Question } \\
\text { No }\end{array}$} & Preferences & 1 & 2 & 3 & 4 & 5 & \multirow{2}{*}{ WA Score } \\
\cline { 2 - 7 } \\
\hline 1 & 0 & 2 & 13 & 20 & 9 & & \\
\hline 2 & 3 & 7 & 19 & 11 & 5 & 9.4666667 & 3 \\
\hline 3 & 2 & 7 & 19 & 12 & 3 & 9.1333333 & 4 \\
\hline 4 & 0 & 9 & 14 & 9 & 12 & 10.4 & 2 \\
\hline 5 & 2 & 10 & 17 & 12 & 3 & 9.066667 & 5 \\
\hline
\end{tabular}

The Weighted Average or the Weighted Mean is used when the relative importance of the items in a series is not same for all items. In this case, each item is judged based on its relative importance. The 5 questions on work life balance the weighted average was calculated to determine the preference given to each question and the results were as follows:

It has been found that employees regarded the salary and benefits as the most important factor to determine Work life Balance. Employees with high emotional intelligence are high performers with lesser stress and burnout and able to manage a balance between work and family life.[9] The employees that exhibited higher regulation of emotion in the self, and recognition of emotion in others, use of emotion to facilitate performance \& motivate self and expression of emotion in the self. The influence of emotional intelligence on work life balance dimensions was observed to be weakly related but significant, while motivation \& expression of emotions in self and use of emotions to facilitate performance displayed larger influence.

Employees with higher emotional Intelligence were those who were fairly happy with their salary and benefits and had a better work life balance. According to a study done by Madipelli, Sarma \& Chinnappaiah [10] in the Indian Context Employees not satisfied with pay and benefits often result in monotony, frustration and stress towards work and home, which leads to work life imbalance.

Employees gave importance to connection between pay and performance, (i.e appraisals, hikes, incentives)Those who were satisfied with the pay with regard to their performance had abetter work life balance. Employees who scored high in EI also believed that they had higher opportunity for advancement. Employees who had lower EI found their work monotonous, more pressure and no job satisfaction at work and had no work life balance.

The fourth ranking was given to flexibility in working hours. Employees with flexible timing were able to manage their work and family responsibilities effectively there by allowing them to minimize work family conflict and to improve the performance at work \& home. Many studies have suggested that flexible work arrangements would help the employees to attain a better blend between work and non work activities and help the organizations to recruit, retain and motivate their employees [11] identified that flexitime work arrangement reduced late comings, absenteeism, and turnover. The study concluded that flexible time strategy improved employee productivity by minimizing absenteeism, turnover and work family conflict.

The Communication between supervisor or Boss and employee was the last ranked in work life balance scheme of things. Employees at lower levels of management the relationship between emotional intelligences influence on work life balance dimensions was significant however the influence was weak on the other hand at middle and higher levels of a management the relationship was pronounced. The employees higher EI had a positive impact on their WIL balance and vice versa.

Significant differences were found between gender and work interference with personal life, work related factors interfering with personal life and level of risk to an employee's position with the usage of worklife balance programs. All the three variables were higher for men as compared to women. 
Significant differences were found between age and work related factors interfering with personal life. Work related factors interfering with personal life were highest for the $30-40$ years age group, followed by the 40- 50 years age group and was lowest for employees above 50 years

\section{Conclusion}

By conducting this study, it was identified that Work Family Life is an important factor in employee performance and one of the reason for employee turnover. Emotional intelligence has a significant effect on persons work life equation and contributes significantly towards maintaining a proper balance in professional and personal life.

WFC (work family conflict) has serious repercussions on the Employees and therefore on organizations too. So recruiting employees with good EI would be a first step towards reducing this conflict and reducing the turnover. Moreover the demographic changes happening in the workforce need also to be considered by Organizations as their Image is at stake. Now younger and younger people and a large number of women too are entering the workforce so Companies would do well to test their EQ along with IQ as a process of recruitment so as to reduce turnover and have a good working environment.

\section{Limitations And Future Recommendations}

While conducting the research, the first limitation was that it was restricted to only one Organization, discussing only two factors in order to conduct the research. However, in the future, the research could be expanded to more dimensions of the same area. Using the variables in link with new mediating variables can make the research more interesting.

In order to have a balanced workforce both employer and employee need to be willing to bend a little. There should be a clear boundary drawn between work and non work activities. Human Resources and the Senior Management take the initiative and communicate the importance of having a good balance between personal and professional life. The widespread perception that visibility = productivity should be changed. Focus on effectiveness rather than on length of work hours. Have shorter working hours with flexibility by using communication technologies and skilful time-management strategies to boost output. This may not be possible in all industries, so apply the best options to reduce rigidity and monotony in work.

Organizations should come up with effective and efficient work life balance policies and programs and foster an organizational culture that supports the use of available policies. This will help reduce the work - life conflict for employees. Organizations should take initiative to improve the emotional intelligence competencies of their employees as it plays a significant role in the kind of work an employee produces, and the relationship he or she enjoys in the organization and improve individual and organizational performance.

Workshops on work life balance and providing awareness on how to become emotionally intelligent in the work place and consequently in other areas of life can be provided by Organizations for employees to have a happy and satisfied workforce.

\section{References}

[1]. Goleman, D. (1995). Emotional intelligence. New York: Bantam Books, Inc.

[2]. J.M. George,'Emotions and Leadership:The role of Emotional Intelligence,” Human Relations, Vol 53,pp 1027-1055, 2000

[3]. D,Goleman, R. Boyatiz and A. Mckee, primal Leadership: Realizing the power of Emotional Intelligence, Boston, MA:Harvard Business School Press:

[4]. K.S.aw, C.Wong,\&L.J.Song,'The construct and criterion validity of emotional intelligenceand its potential utility for managerial studies.” Journal of applied Psychology.vol89,pp483-496,2004

[5]. T. Sy, \& S.Cote, Emotional Intelligence: A Key ability to succeed in the matrix Organization. Journal of Management Development.vol23,pp437-455,2004

[6]. Jivan Jyoti Maini, Bhawdeep Singh,Parminder Kaur. The Relationship among Emotional Intelligence and Outcome variables: A study on Indian Employees Vision - The Journal of Business perspective

[7]. Gupta, Sachin, Research Paper on Emotional Intelligence and Work Life Balance of Employees in the Information Technology Industry (February 13, 2014).

[8]. https://www.linkedin.com/pulse/how-measure-your-emotional-intelligence-daniel-goleman.

[9]. http://papers.ssm.com/scl3/papers.cfm?abstract_id=289 100

[10]. Sarita Madepalli, V.S.Veluri Sarma, Y. Shinnappaiah. "Factors Causing Worklife Imbalance among working women-A Study on School Teachers. Indian Journal of Industrial Relations. Vol48 Issue 4, 2013

[11]. Christensen KE,Staines GL,(1990) Flexitime: A viable solution to Work Family Conflict. Journal of Faily Issues.11(4) : 455-476.

[12]. http://www.ijmbr.org/article_1_a89bbb153e811c311fafa62bdd39b486.pdf, 13-07-16

[13]. https://www.linkedin.com/pulse/how-measure-your-emotional-intelligence-danielgoleman?trk=li_fb_cmktg_injun_1\&sf27268284=1

[14]. http://www.ijmbr.org/article_1_a89bbb153e811c311fafa62bdd39b486.pdf, 13-07-16

[15]. http://www.Indian Journals.com: Kundu Dipesh ,Exploring the Relationship between Emotional Intelligence and Leadership Behaviour among Sole Proprietors in India 